\title{
On the Usage of Precision Timing Detectors in High Rate and High Pileup Environments
}

\author{
Adi Bornheim* \\ Caltech, 1200 E California Blvd, Pasadena, CA, USA \\ E-mail: bornheimehep. caltech.edu
}

\begin{abstract}
High energy particle collider experiments are facing ever more challenging conditions, operating at todays accelerators capable of providing instantaneous luminosities of $10^{34} \mathrm{~cm}^{-2} \mathrm{~s}^{-1}$ and above. The high center of mass energy, the large number of simultaneous collision of beam particles in the experiments and the very high repetition rates of the collision events pose huge challenges. They result in extremely high particle fluxes, causing very high occupancies in the particle physics detectors operating at these machines. A precise timing information with a precision of around $10 \mathrm{ps}$ and below is seen as a major aid in the reconstruction of the physics events under such challenging conditions. In this paper I discuss applications of precision timing in high pile-up conditions and review the efforts of the LHC collaborations to augment the timing performance of their detectors during future upgrade campaigns. Different detector technologies allowing precision timing measurements will be discussed and their potential benefit for a holistic event reconstruction will be illustrated.
\end{abstract}

The 25th International workshop on vertex detectors

September 26-30, 2016

La Biodola, Isola d'Elba, ITALY

${ }^{*}$ Speaker. 


\section{Introduction}

Advances in sensor technology and readout electronics have given access to timing precisions on the order $10 \mathrm{ps}$ for the detection of high energy particles. While the focus of the hardware development is moving towards the 1 ps domain and beyond, technologies with order 10 ps resolution are now considered for large scale applications. In particular the challenges of current and future high luminosity hadron colliders may be best addressed by enhancing the timing performance of the detectors to the level of $10 \mathrm{ps}$. To fully exploit the physics potential of the luminosity upgrade of the Large Hadron Collider (LHC), the High Luminosity LHC (HL-LHC), the experiments ATLAS and CMS are investigating precision timing upgrades of their respective detectors. The ALICE collaboration has spearheaded precision timing technologies at LHC and LHCb is investigating possible upgrades for time-of-flight based particle identification. The physics goal of the ATLAS and CMS collaborations at HL-LHC is to precisely study Higgs boson production, decay kinematics and rates as well as the measurement of the Higgs self coupling. This will be a crucial additional test of the SM electroweak symmetry breaking sector. Searches for physics beyond the standard model are also part of the program. To achieve these goals an integrated luminosity of at last $3 \mathrm{ab}^{-1}$ has to be accumulated over a time span of 10 years. The detector performance needs to be maintained at a level of the LHC Run I data. The instantaneous luminosity will be at the level of $10^{35} \mathrm{~cm}^{-2} \mathrm{~s}^{-1}$ [1]. This requires the operation of the HL-LHC at beam intensities that will result in 200 simultaneous proton-proton collisions (PU) per bunch crossing.

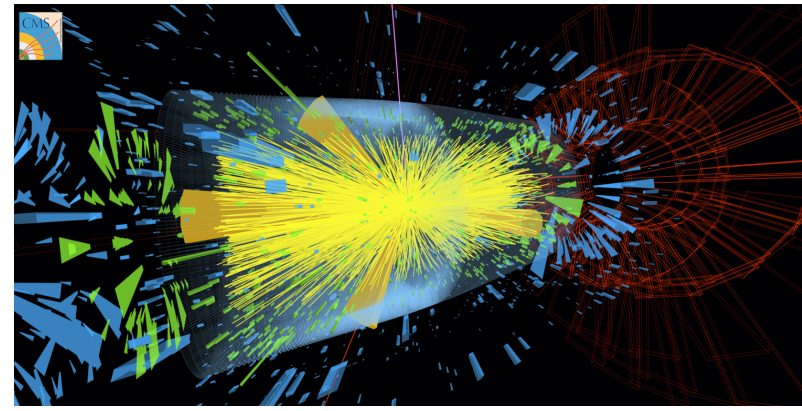

Figure 1: Simulated event of a Higgs Boson, produced in association with two jets, decaying into two photons. The simlated event is embedded in 200 PU events. Associating the final state objects of the Higgs decay to each other via their common production vertex is very challenging in this environment.

To maintain the physics performance of the detectors at HL-LHC the granularity and speed of the detectors as well as the readout and trigger electronics will be improved. Enhancing the timing performance of the detectors will give an additional handle to disentangle the superposition of the hard interaction with 200 PU events. In Fig. 1 we show a simulated event in the CMS detector where a Higgs Boson, produced in association with two jets, decays into two photons. The event is embedded in $200 \mathrm{PU}$ events which dominate the event display.

\section{Physics use cases for precsion timing at HL-LHC}

The key challenge at HL-LHC is the extreme density of particles. This challenges the event reconstruction since the association of information from different subdetectors, eg. matching of vertices to calorimeter clusters, becomes much less reliable and the combination of subdetector information, eg. the combination of multiple tracks to form an event vertex or the combination of multiple calorimeter clusters to form a jet, prone to confusion. In the following we discuss the 
challenges of the event reconstruction in a high pile-up environment with examples from the CMS detector.

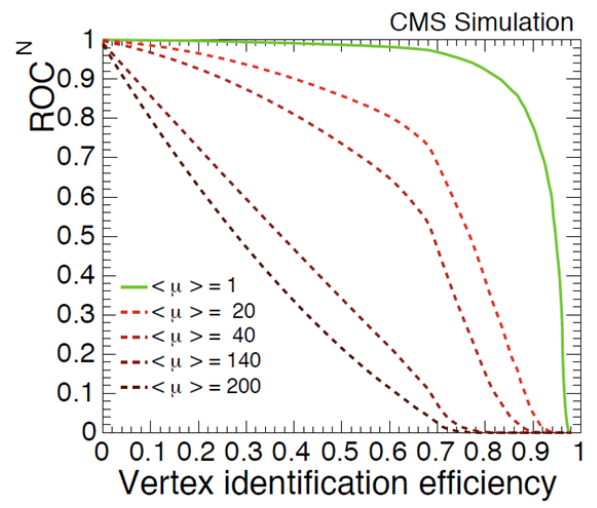

Figure 2: Vertex identification efficiency for $H \rightarrow \gamma \gamma$ events in the CMS detector for different PU scenarios [2]. The vertex identification algorithm uses the summed $p_{T}$ of all the tracks from each identified vertex as one of the main variables to find the vertex of the hard proton-proton collision vertices. In very high PU conditions, as expected at HLLHC, this strategy is not very efficient any more. The promotion of PU vertices to higher $\sum p_{T}^{2}$ requires additional information to determine the primary vertex in the event.

In Fig.2 we show the efficiency of assigning the two photons of a Higgs Boson decay to the correct vertex for different PU scenarios from 0 PU to 200 PU events, the current upper range of the expected HL-LHC operational conditions. The vertex identification algorithm largely depends on the summed transverse momentum of the tracks from the vertices of all proton collisions within one bunch crossing. This algorithm does not yield a reliable determination of the di-photon vertex any more.

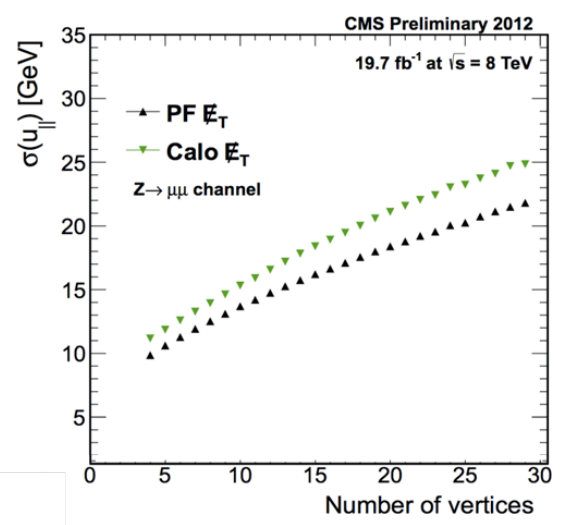

Figure 3: Missing transverse energy $\left(\mathscr{E}_{T}\right)$ resolution as a function of the PU. Each PU event adds $3 \mathrm{GeV}$ in quadrature to the $\not E_{T}$ resolution [3], stemming from PU tracks and clusters assigned to the hard vertex. The particle flow (PF) based event reconstruction improves the performance only slightly by combining tracks and calorimeter clusters.

Similarly affected is the determination of the missing transverse momentum $\left(\mathscr{E}_{T}\right)$ and the proper determination of the jet energy. Fig. 3 shows the scaling of the $\mathscr{E}_{T}$-resolution as a function of the PU which adds about $3 \mathrm{GeV}$ in quadrature for each PU event as energy flow from PU events gets wrongly assigned to the hard interaction vertex. With precision timing the association of particles to primary vertices would be more efficient and mis-assignment from PU activity could be reduced. This is expected to recover the loss in resolution performance up to the PU rates of HL-LHC. Fig. 4 shows the jet energy resolution which deteriorates in the high PU environment as calorimeter clusters and tracks from PU events get assigned to a jet from the hard vertex. At $p_{T}=40 \mathrm{GeV}$ around $75 \%$ of the energy flow in the jet cone are from PU vertices. One third of the hadronic activity is carried by neutral particles and a large fraction by low $p_{T}$ particles for which the momentum resolution is poor in the forward direction. Precise timing will allow to associate neutral calorimeter clusters to the proper vertex, rejecting activity from PU vertices. 


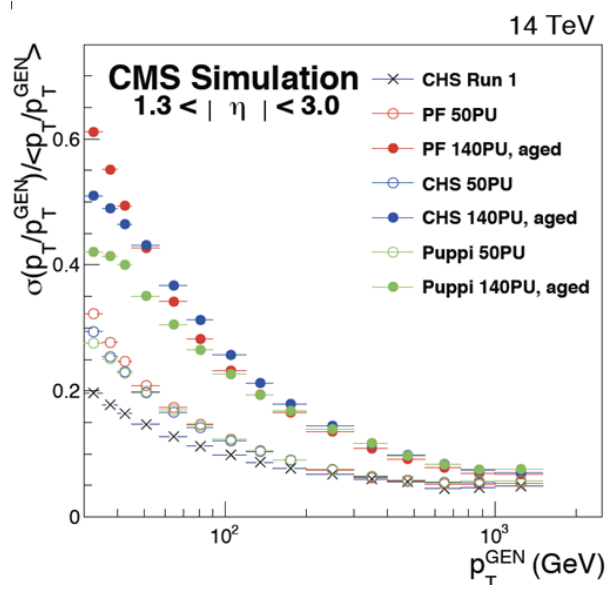

Figure 4: Jet Energy Resolution (JER) as a function of the transverse momentum $p_{T}$ of the jet for different PU scenarios. The Charged Hadron Subtraction (CHS), Particle Flow (PF) and the Pileup per Particle Identification (PUPPI) reconstruction methods are compared.

While high granularity calorimeters and tracking devices as well as advanced reconstruction algorithms can mitigate the performance degradation, the level of early LHC data, which was virtually unaffected by PU, is not restored.

Adding precision timing information is expected to improve the performance, arriving at a truly holistic event reconstruction exploiting the maximum information available. Timing measurements for high energy photons require a calorimetric device which makes photon vertexing the key application of precision timing calorimeters. Precise timing of tracks is particularly useful to improve the assignment of tracks to vertices they originate from. In Fig. 5 we show the impact of precision timing for photons, tracks and the combination of both on the $H \rightarrow \gamma \gamma$ mass resolution [4]. The mass resolution is affected by misidentifying the true event vertex as well as the photon energy resolution of the ECAL. It is sufficient to reconstruct the di-photon vertex within about $1 \mathrm{~cm}$ of the true vertex. Below this value the impact on the mass resolution is negligible with respect to the photon energy resolution. For about $50 \%$ of the di-photon events the vertex can be reconstructed from the photon timing alone. With precise timing information for the tracks in the events the vertex reconstruction performance can be restored to the level achieved at LHC Run1.

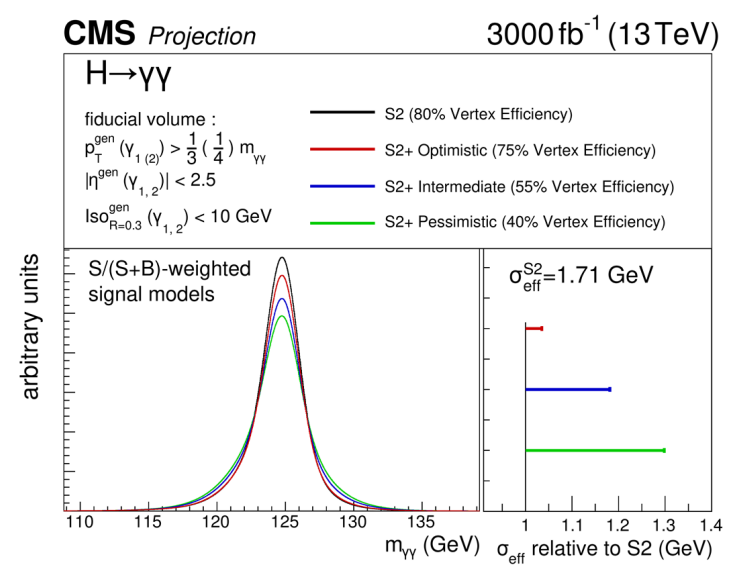

Figure 5: Impact of precision timing for photons, tracks and the combination of both on the $H \rightarrow \gamma \gamma$ mass resolution [4]. In the scenario shown, S2, the detector performance is kept as for LHC Run 2, while experimental systematic uncertainties are scaled down with luminosity until a lower bound based on the expected future achievable uncertainties is reached. The pessimistic scenario assumes a vertex finding algorithm as in Run1 (green), the intermediate scenario assumes calorimeter precision timing (blue) and the optimistic scenario assumes calorimeter and track based precision timing (red).

In Fig. 6 we illustrate the basic principle of associating physics objects with the help of precision timing. Here, a di-photon final state is depicted, assuming a timing precision of around $30 \mathrm{ps}$ for each of the two photons. With the position determination of the photons from the calorimeters, which has a precision of better than one $\mathrm{mm}$, and the constraint from the $\mathrm{x}$ - and y-position of the 
beam axis we are left with two unknowns, the vertex z-position and the time of the collision. With the available information the z-position and time of the collision can be calculated analytically. For many-body final states the same concept applies, albeit the determination of the vertex has to be performed numerically.

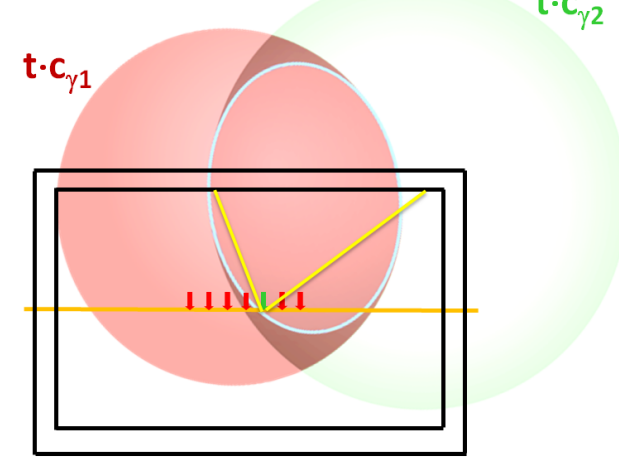

Figure 6: Illustration of the di-photon vertex reconstruction from the position and time of arrival of two photons. The sketch represents a view on a collider experiment detector with two calorimeter deposits. The spheres depict the event horizont of the two deposits for a given time. The two spheres, together with the beam axis shown as an orange line will have a single intersection point inside the detector which can be referred to as the timing vertex. Associating it with the track vertices (green and red arrows) will allow to discard PU vertices not associated to the calorimeter deposits.

These examples illustrate how precision timing could be used to improve the physics object and event reconstruction in a high PU environment. The usage of precision timing is also studied for the lower level reconstruction such as the clustering of individual calorimeter cells and the reconstruction of tracks from individual hits [5].

\section{Calorimeter precision timing}

Calorimeters can exploit the temporal coherence of electromagnetic showers to achieve very good timing performance. In Fig. 7 we show the time resolution measured with a micro-channel plate photomultiplier (MCP-PMT) in an electromagnetic shower.
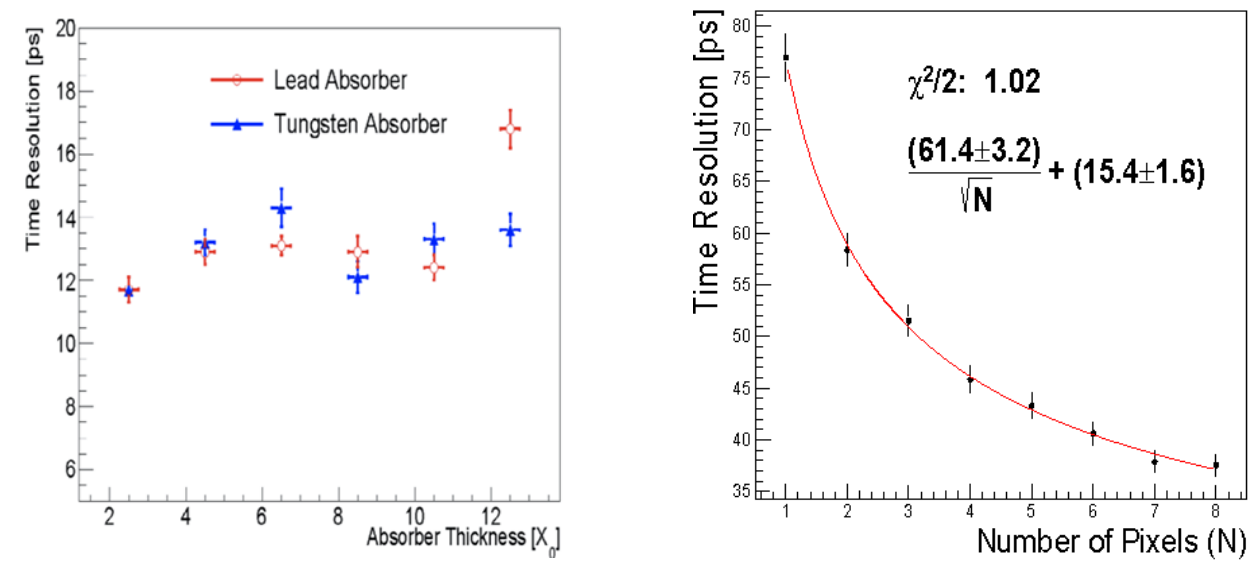

Figure 7: Time of arrival of a electromagnetic shower at different depth in a calorimeter (left). The timing precision at around $12 \mathrm{ps}$ is independent of the shower depth. Time of arrival measurement with a pixelated MCP (right). By combining the information of multiple pixels with a single channel resolution of around 75 ps yields a system performance of around 35 ps.

A timing precision of around $12 \mathrm{ps}$ is measured for electrons of $32 \mathrm{GeV}$ with respect to a Photek 240 MCP-PMT serving as a reference counter, registering the time of arrival on the incoming electron before it interacts with any absorber. The timing precision is largely independent of the shower 
depth and between two different absorber materials. This illustrates that the entire shower can be exploited to determine the time of arrival of the incoming particle. In particular this allows to integrate the large primary signals induced eg. in scintillation detectors or to place multiple sensors in the same shower such as in sampling calorimeters. In Fig. 7 (right) we show the timing performance measured with a pixelated MCP, combining the measurement from 8 independent measurements. A precision of $35 \mathrm{ps}$ is measured combining single channels with a resolution of $75 \mathrm{ps}$ [6].
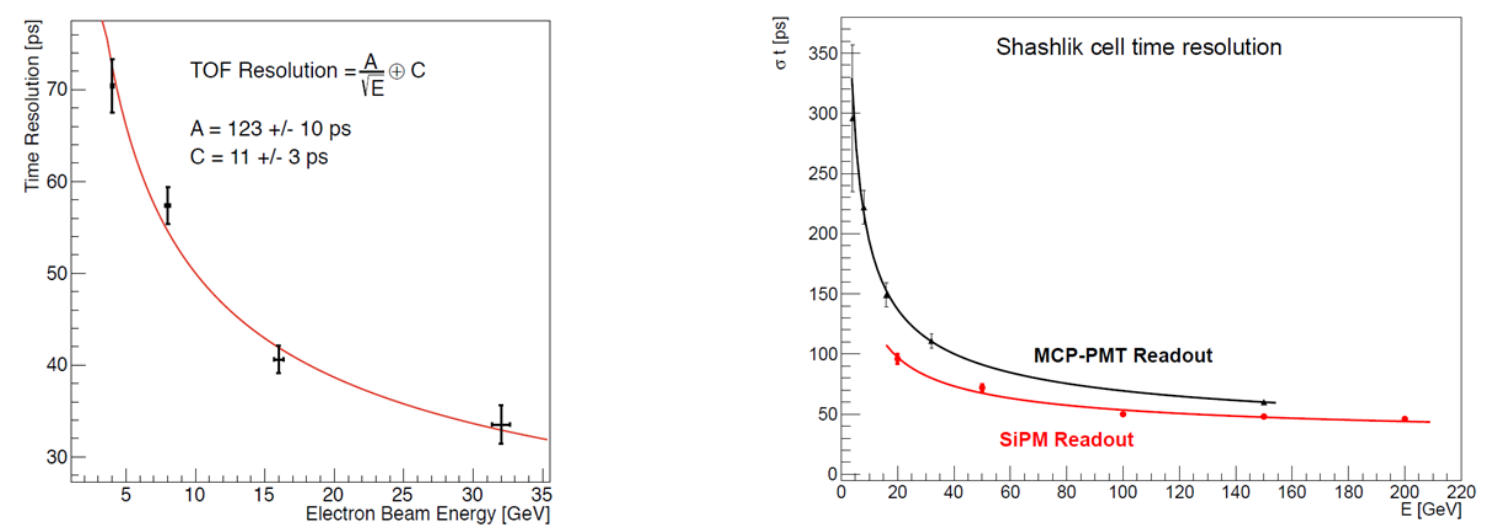

Figure 8: Timing resolution of a LYSO cube of $1.7 \mathrm{~cm}$ size (left) and a LYSO/W sampling calorimeter cell (SH) with wave length shifting capillary readout (right). For large signals a timing resolution of $32 \mathrm{ps}$ and $48 \mathrm{ps}$ is achieved. The SH cells simultaneously provides an energy resolution of $10 \% \sqrt{E} \oplus 1 \%$ [7].

In Fig. 8 we show timing results from a solid LYSO cube and of a LYSO/W sampling calorimeter (SH). An MCP-PMT was used as a photo detector for the LYSO cube and both MCP-PMTs and SiPMs for the SH cell. A resolution of 32 ps was measured for the LYSO cube with $32 \mathrm{GeV}$ electrons and $48 \mathrm{ps}$ for the SH cell with $200 \mathrm{GeV}$ electrons [8]. The output signal of the photo detectors were not amplified in these setups, which limits the timing performance towards low signal amplitudes due to the noise of the readout electronics. The experimental results in Fig. 8 demonstrates that a timing resolution of a few $10 \mathrm{ps}$ can be achieved with scintillator based calorimeters, leading to the decision of the CMS Collaboration to improve the timing performance of the CMS ECAL in the Phase II upgrade for HL-LHC.

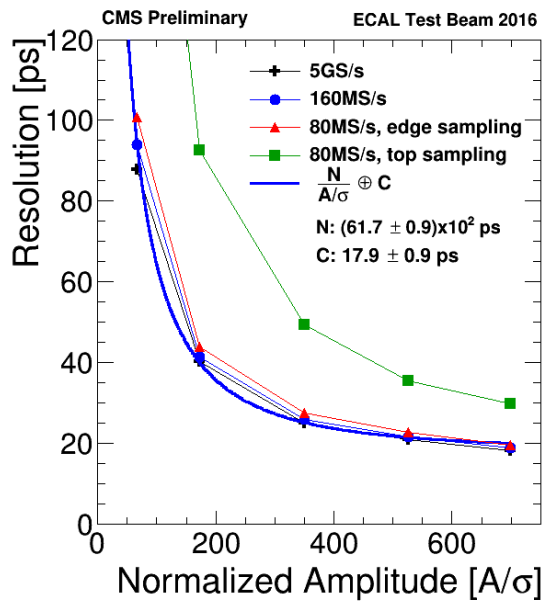

Figure 9: Measured timing resolution of the CMS ECAL with a prototype of the electronics envisioned for the Phase II upgrade [9]. A time resolution of about 20 ps was achieved for high energy electromagnetic deposits. Different digitization rates are emulated by only using a fraction of the available $5 \mathrm{GS} / \mathrm{s}$. It is demonstrated that a sampling rate of 160 $\mathrm{MS} / \mathrm{s}$ will yield the best possible timing resolution based on the existing APD photo sensors.

In Fig. 9 we show the results of recent test beams performed in preparation of the HL-LHC upgrade 
of the CMS electromagnetic calorimeter (ECAL) barrel. For large signals from the photo detector a timing resolution of about $20 \mathrm{ps}$ is achieved down to energies of about $10 \mathrm{GeV}$ [9]. A resolution of better than $30 \mathrm{ps}$ will be maintained for typical photons stemming from Higgs decays for the HL-LHC data taking. The endcap of the CMS calorimeter detector will be replaced for the Phase II upgrade. The technology choice of the CMS Collaboration is a sampling calorimeter with silicon sensors in the electromagnetic and front third of the hadron calorimeter section (HGCAL) and scintillator in the remaining portion of the hadron calorimeter (BC). Fig. 10 shows test beam measurements with a single silicon pad. With the large primary signal induced by high energy electrons a timing precision of better than 20 ps can be achieved. This suggests that such good performance can be reached with the upgraded CMS endcap calorimeter [10]. The precision timing upgrades

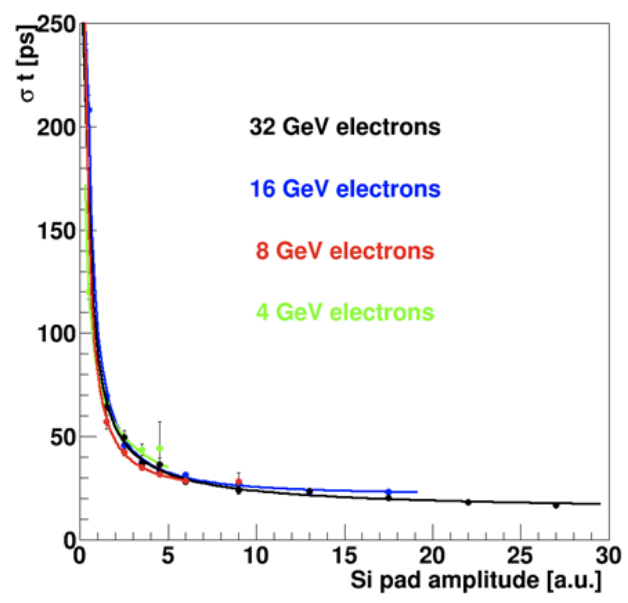

Figure 10: Timing resolution measured with a single silicon pad with a size of $1 \mathrm{~cm}^{2}$, placed in an electromagnetic shower induced by electrons with energies between $4 \mathrm{GeV}$ and $32 \mathrm{GeV}$. Details on this measurement can be found in [11].

of the CMS calorimeters take advantage of the necessity to replace the endcap calorimeter and upgrade the barrel ECAL. These devices will however only achieve the required timing resolution of a few $10 \mathrm{ps}$ for energy deposits of $10 \mathrm{GeV}$ and above. To extend the precision timing capabilities of the detectors to a lower momentum range, a necessity to fully exploit the potential of precision timing, different technologies have to be considered.

\section{Precision timing for tracks}

Charged particle tracking detectors are relying predominantly on silicon sensors. High detection efficiency for charged particles, high radiation tolerance and an increasing level of integration of front end electronics and sensors into the chip design are important for tracking detectors. The timing precision of silicon detectors is limited by landau fluctuations. Various sensor and detector technologies may be considered to achieve a timing performance in the range of $10 \mathrm{ps}$ for charge tracks.

\subsection{Silicon sensors without gain.}

A timing precision of around $200 \mathrm{ps}$ is achievable in a large scale system based on silicon sensors without gain as eg. demonstrated by the the NA62 silicon tracker [12, 13]. As discussed in the case of precision timing calorimeters in Sec. 3, the timing performance of a detector system 
can be improved by using multiple measurements on the same physics object such as a calorimeter cluster or a track. Combining the information allows to overcome the limitations of single sensor performance. This approach is taken successfully by the MEG-II experiment [14], using scintillator plates with SiPM readout, reaching 30 ps with 8 sensors. As demonstrated in Fig. 7, a square-root like scaling can be assumed when estimating the timing performance of a system combining multiple measurements. Large silicon tracking devices as the ones from the CMS and ATLAS detectors have typically between 10 and 20 layers of sensors. With a single layer performance of $200 \mathrm{ps}$ this may lead to a combined performance of a few $10 \mathrm{ps}$ if all layers had precision timing capabilities. The requirements to minimize the data transfer bandwidth, power consumption and cooling needs of the tracking devices however pose significant hurdles to such an approach with todays technologies. While this is not pursued for the HL-LHC upgrades of CMS and ATLAS it may well be an option for future experiments.

Instead, the LHC collaborations currently investigate the possibility of inserting a precision timing device between the tracking detector and the calorimeters which would measure the time of arrival of tracks with a precision on the order of $10 \mathrm{ps}$. Tracks would be extrapolated into this timing layer where it is associated to a channel in the timing layer which provides a unique time stamp to each track. The granularity of the timing detector is chosen such that the hit occupancy per channel remains below $10 \%$ to limit the instances of multiple hits. From MC simulations for the HL-LHC upgrade of the CMS detector it is estimated that a granularity of $1 \mathrm{~cm}^{2}$ would be sufficient in the barrel part of the detector and the outer part of the endcap, increasing to $0.5 \mathrm{~cm}^{2}$, possibly down to $0.1 \mathrm{~cm}^{2}$ in the most forward region at $\eta=4$. The CMS collaboration is investigating options to equip the entire rapidity range up to $\eta=3$ with a timing layer [15], while ATLAS is studying options which would focus on the forward region from $\eta=2.5$ possibly up to $\eta=5.0$, referred to as High Granularity Precision Timing Device (HGPTD) [16]. A number of technologies are considered for such a timing layer in the ATLAS and CMS experiments.

\subsection{Deep-depleted Avalanche Photo Diodes.}

The principle limitation of the timing performance of silicon detectors can be mitigated by introducing gain to the sensor. One approach being followed is the usage of deep-depleted Avalanche Photo Diodes (dAPD), equipped with a mesh screen to uniformize the electric field inside the silicon. Field inhomogeneities in the sensor are suspected to be a reason limiting the performance of existing silicon based tracking devices to 200 ps [17]. Prototypes of such sensors have been tested and show promising results. CMS is investigating the usage of dAPD for a possible endcap timing layer. These devices may maintain good performance even at a size of $1 \mathrm{~cm}^{2}$ which would be advantageous to cover larger areas.

\subsection{Low-gain Avalanche Diodes.}

An alternative approach to enhance the timing performance for silicon devices is the usage of thin, low gain avalanche detectors (LGAD) $[18,19]$. These sensors have a gain layer similar to APDs but with a modified doping profile. A modest gain on the order of a few times 10 is realized in a thin sensor with a thickness of $50 \mu \mathrm{m}$. The thin layer ensures a fast signal rise time and typically ensures better radiation resistance. A timing performance of $26 \mathrm{ps}$ has been reported for a 
single sensor [20]. And arrangement of three sensor layers achieved a combined timing resolution of $15 \mathrm{ps}$. This sensor technology is the choice of the ATLAS collaboration for their HGPTD. CMS is considering this technology as well for their endcap timing layer. The size of the sensors being tested to date is a few $\mathrm{mm}^{2}$. The performance is expected to degrade for larger senors of eg. $1 \mathrm{~cm}^{2}$. Covering the endcap regions of either the CMS or ATLAS detectors requires an active area of several $\mathrm{m}^{2}$. R\&D is ongoing to develop a full detector concept for the HL-LHC upgrades of the two detectors.

\subsection{LYSO based detectors with SiPM readout.}

As was discussed in Sec. 3, LYSO is well suited for precision timing measurements due to its very high light yield and very fast signal rise time [8,21]. The energy deposited in $1 \mathrm{~mm}$ of LYSO by a charged particle is about $1 \mathrm{MeV}$ which results in the creation of about 30000 scintillation photons. It has been demonstrated that $3 \mathrm{~mm}$ of LYSO crystal, read out with an SiPM, can achieve a timing resolution of better than 20 ps for charged particles [22]. The CMS collaboration is investigating the possibility of a timing layer consisting of LYSO tiles with a size of $1 \mathrm{~cm}^{2}$ and a thickness of $3 \mathrm{~mm}$, read out with SiPMs. It would cover the front the the CMS ECAL barrel and be installed on the mechanical support structure of the silicon tracker. It would cover an area of about $40 \mathrm{~m}^{2}$.

\subsection{Micro-channel plate devices.}

MCP based devices have shown very good timing performance for single charged particles. In laboratory setups MCP-PMTs are often used as reference timing detectors. In Fig. 11 we show the timing performance of two commercial MCP-PMTs, measuring a single device timing resolution of around $7 \mathrm{ps}$ [23]. A charged particle passing through the sensor window results in the emission of Cherenkov photons which subsequently get detected by the MCP, operating as a photo detector. The prompt emission of the Cherenkov photons and the small distance of which they get emitted maintains the very small transit time jitter of the device. Charged particles however also induce direct ionization in the MCP layer with subsequent amplification equivalent to the detection of a photoelectrons from the photo cathode layer, referred to as secondary emission mode. Extensive

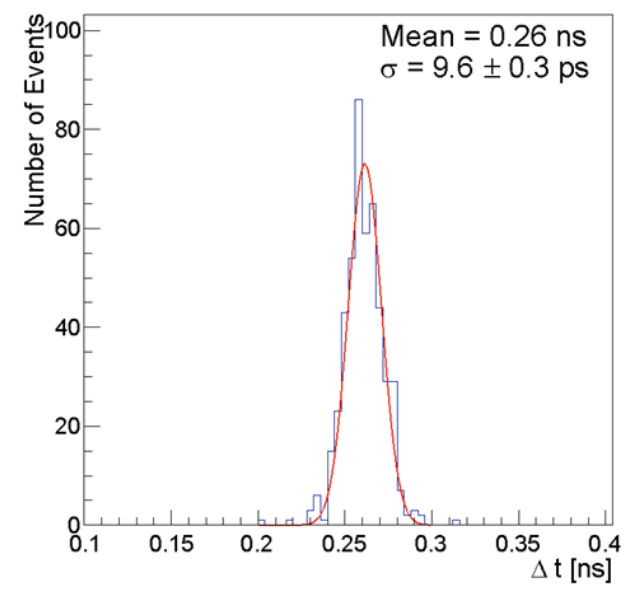

Figure 11: Differential timing distribution between two Photek 240 MCP-PMT measured with $120 \mathrm{GeV}$ protons. No absorber was used in the beam line. The protons pass through both MCP-PMT as a minimum ionizing particle (MIP). The differential time distribution was measured to be around $10 \mathrm{ps}$ which corresponds to a single device resolution of $7 \mathrm{ps}$. The readout system has a resolution of around 5 ps [23]. The MCPs and the DAQ used in this setup were used as reference timing detector in all the results presented in this paper.

R\&D has been carried out to explore if a timing layer for CMS could be realized with MCPs op- 
erating in secondary emission mode. The progress made for large area picosecond photo detectors (LAPPD) [24] provides a very solid basis for this effort. While the HL-LHC upgrades may not allow for sufficient time to mature the technology it remains a very promising technology for the future beyond HL-LHC.

\section{Further Applications of Precision Timing}

The timing resolutions of the order of $10 \mathrm{ps}$ and the size of current hadron collider experiments allows to perform time-of-flight (TOF) based particle identification (PI) which has been an integral part of B-factory experiments since long. The ALICE Collaboration has demonstrated that TOF based PI is viable in the LHC environment [25]. The LHCb collaboration is investigating an upgrade option similar to the technology used at the B-factories, based on a large quartz plate in which charged particles emit Cherenkov light [26]. The light is guided via internal reflection onto a photo detector. The design target is a resolution of 15 ps per track which would result in a K- $\pi$ separation of about $3 \sigma$ for particle momenta of $10 \mathrm{GeV}$, above which a ring-imaging Cherenkov detector is used. A timing performance of around 100 ps was achieved with 2015 test beam results and a significant improvement was seen in 2016 test beams [27]. A single track time resolution of the order of a few $10 \mathrm{ps}$ in CMS or ATLAS would allow to be extend the momentum range of the de/dx based PI significantly.

\section{Summary}

Precision timing can be a powerful handle to mitigate pile-up effects at HL-LHC. A wide range of sensor and detector technologies allow to reach timing measurement precision at the level of 10 ps. There is very active R\&D towards the HL-LHC upgrades to enhance the timing performance of the existing detectors and to possibly add dedicated precision timing detectors. Future experiments will be able to implement precision timing technologies in all sub-detector components which will greatly enhance the capability to reconstruct events in very dense environments such as high pileup hadron collisions. Precision timing may benefit the performance of particle physics detectors in the sub-detector as well as the full event reconstruction, making it a valuable additional tool in achieving a holistic event reconstruction, challenging the traditional subdetector division.

\section{References}

[1] L. Rossi, and O. Brüning, High Luminosity Large Hadron Collider A description for the European Strategy Preparatory Group, CERN-ATS-2012-236

[2] CMS Collaboration, Initial Report of the Fast Timing Working Group, CERN-CMS-DP-2016-008

[3] CMS Collaboration, MET performance in $8 \mathrm{TeV}$ data, CMS-PAS-JME-12-002

[4] J. Bendavid, Precision Timing: CMS Plans and Performance Studies, ECFA High Luminosity LHC Experiments Workshop 2016, https://indico.cern.ch/event/524795/

[5] M. Fiorini, 4D fast tracking for experiments at the High Luminosity LHC, VERTEX 2016, these proceedings

[6] A. Apresyan et al, Direct tests of a pixelated microchannel plate as the active element of a shower maximum detector, NIM-A, http://dx.doi.org/10.1016/j.nima.2016.05.015 
[7] B. Cox on behalf of The Shashlik Collaboration, The Shashlik Calorimeter, a LYSO/W plate Calorimeter for Precision EM Calorimeter in the High Luminosity LHC environment, to appear in the proceedings of ICHEP 2016, Chicago, USA

[8] D. Anderson et al, On timing properties of LYSO-based calorimeters, NIM-A, http://dx.doi.org/10.1016/j.nima.2015.04.013

[9] A. Bornheim, for the CMS Collaboration, Design studies for the Phase II upgrade of the CMS Barrel Electromagnetic Calorimeter, to appear in the proceedings of TWEPP 2016

[10] A. Apresyan, for the CMS Collaboration, Investigation of Fast Timing Capabilities of Silicon Sensors for the CMS High Granularity Calorimeter at HL-LHC, proceedings of IEEE NSS/MIC 2016

[11] A. Apresyan et al, Test Beam Studies Of Silicon Timing for Use in Calorimetry, NIM-A, doi:10.1016/j.nima.2016.04.031

[12] M. Fiorini, Operational experience with the NA62 Gigatracker, VERTEX 2016, these proceedings

[13] M. Noy, Chip Development for High Time Resolution Silicon Detectors, VERTEX 2016, these proceedings

[14] M. De Gerone et al. ,A high resolution Timing Counter for the MEG II experiment, NIM-A, http://dx.doi.org/10.1016/j.nima.2015.11.022

[15] The CMS Collaboration, CMS Phase II Upgrade Scope Document, CERN-LHCC-2015-019

[16] The ATLAS Collaboration, ATLAS Phase-II Upgrade Scoping Document, CERN-LHCC-2015-020

[17] S. White, Fast Timing Detector R\&D for the HLLHC era, Frontier Detectors for Frontier Physics 13th Pisa Meeting on Advanced Detectors, Elba 2015, https://agenda.infn.it/getFile.py/access?contribId=23\&sessionId=12\&resId=0\&materialId=slides\&confId=8397

[18] G. Pellegrini et al, Technology developments and first measurements of Low Gain Avalanche Detectors (LGAD) for highenergy physics applications, http://dx.doi.org/10.1016/j.nima.2014.06.008

[19] S. Meroli, D. Passeria, and L. Servoli, Energy loss measurement for charged particles in very thin silicon layers, http://dx.doi.org/10.1088/1748-0221/6/06/P06013

[20] N. Cartiglia et al, Beam test results of a 15 ps timing system based on ultra-fast silicon detectors, https://arxiv.org/ 1608.0881, to be published in NIM-A

[21] A. Bornheim, Precision Timing Calorimetry for High Energy Physics, Frontier Detectors for Frontier Physics 13th Pisa Meeting on Advanced Detectors, Elba 2015, http://dx.doi.org/10.1016/j.nima.2015.11.129

[22] A. Benaglia et al,Detection of high energy muons with sub-20 ps timing resolution using L(Y)SO crystals and SiPM readout, http://dx.doi.org 10.1016/j.nima.2016.05.030

[23] A. Ronzhin et al, Study of the timing performance of micro-channel plate photomultiplier for use as an active layer in a shower maximum detector, NIM-A, http://dx.doi.org/10.1016/j.nima.2015.06.006

[24] H. Frisch et al, A Brief Technical History of the Large-Area Picosecond Photodetector (LAPPD) Collaboration, https://arxiv.org/abs/1603.01843

[25] A. Alici, Status and performance of the ALICE MRPC-based Time-Of-Flight detector, JINST 7 P10024, http://dx.doi.org/10.1088/1748-0221/7/10/P10024

[26] K. Fohl, for the LHCb Collaboration, TORCH - an Innovative High-Precision Time-of-Flight PID Detector for the LHCb Upgrade, CERN-LHCb-PROC-2015-001

[27] T. Gys, The TORCH detector R\&D: Status and Perspectives, RICH2016, https://indico.cern.ch/event/393078/contributions/2195346/ 